\title{
Decreased in vivo glutamate/GABA ratio correlates with the social behavior deficit in a mouse model of autism spectrum disorder
}

\author{
Gaeun Park ${ }^{1,2 \dagger}$, Se Jin Jeon ${ }^{3 \dagger}$, In Ok Ko ${ }^{4}$, Ji Hwan Park ${ }^{5}$, Kyo Chul Lee ${ }^{4}$, Min-Sik Kim ${ }^{5}$, Chan Young Shin ${ }^{3}$, \\ Hyeonjin $\mathrm{Kim}^{6}$ and Yong-Seok Lee ${ }^{1,2,7,8^{*}}$ (D)
}

\begin{abstract}
To diagnose autism spectrum disorder (ASD), researchers have sought biomarkers whose alterations correlate with the susceptibility to ASD. However, biomarkers closely related to the pathophysiology of ASD are lacking. Even though excitation/inhibition (E/I) imbalance has been suggested as an underlying mechanism of ASD, few studies have investigated the actual ratio of glutamate (Glu) to $\gamma$-aminobutyric acid (GABA) concentration in vivo. Moreover, there are controversies in the directions of E/I ratio alterations even in extensively studied ASD animal models. Here, using proton magnetic resonance spectroscopy ( $\left.{ }^{1} \mathrm{H}-\mathrm{MRS}\right)$ at 9.4T, we found significant differences in the levels of different metabolites or their ratios in the prefrontal cortex and hippocampus of $\mathrm{Cntnap2^{-/ }}$ mice compared to their wild-type littermates. The Glu/GABA ratio, N-acetylaspartate (NAA)/total creatine (tCr) ratio, and tCr level in the prefrontal cortex were significantly different in Cntnap2 ${ }^{-1-}$ mice compared to those in wild-type mice, and they significantly correlated with the sociability of mice. Moreover, receiver operating characteristic (ROC) analyses indicated high specificity and selectivity of these metabolites in discriminating genotypes. These results suggest that the lowered Glu/GABA ratio in the prefrontal cortex along with the changes in the other metabolites might contribute to the social behavior deficit in Cntnap2 $2^{-1}$ mice. Our results also demonstrate the utility of ${ }^{1} \mathrm{H}-\mathrm{MRS}$ in investigating the underlying mechanisms or the diagnosis of ASD.
\end{abstract}

Keywords: Autism spectrum disorder, E/l balance, Glu/GABA, Creatine, Sociability, Magnetic resonance spectroscopy

\section{Introduction}

Autism spectrum disorder (ASD) is a neurodevelopmental disorder that exhibits a range of behavioral symptoms, such as restricted interests and repetitive behaviors, and impaired social interactions [1]. For a swift medical intervention for ASD patients, diverse approaches to diagnose ASD have been undertaken that have lowered the minimum age for ASD diagnosis to 18 months from birth

\footnotetext{
*Correspondence: yongseok7@snu.ac.kr

${ }^{\dagger}$ Gaeun Park and Se Jin Jeon contributed equally to this work

1 Department of Biomedical Sciences, BK21 Four Biomedical Science

Program, Seoul National University College of Medicine, Seoul, Republic of Korea

Full list of author information is available at the end of the article
}

[2]. However, finding prominent biomarkers for ASD remains the focus of clinical professionals and researchers, as early intervention can successfully alleviate phenotypical severity before the pervasive symptoms of ASD may arise $[3,4]$.

Classically, ASD can be diagnosed with the patients' behavioral abnormalities as they are unable to react to socially relevant stimuli [5]. Metabolic biomarkers, such as blood serotonin levels, have been examined, as many metabolic disorders are possibly associated with autistic phenotypes [6, 7]. Non-invasive, but more direct biomarkers of ASD using neuroimaging have recently been identified. Functional connectivity between different regions has been observed and used to diagnose ASD [8]. 
The theta-gamma frequency in the frontal lobe is high in children with ASD, but not in typically developing (TD) groups, as shown by electroencephalogram (EEG) analytics [9]. Magnetic resonance imaging (MRI) or positron emission tomography (PET) facilitates in discerning subtle changes in region-specific brain volume, often shown in neurodevelopmental disorders [10]. Proton magnetic resonance spectroscopy $\left({ }^{1} \mathrm{H}-\mathrm{MRS}\right)$ can directly observe the abundance of metabolites in specific brain regions and has been suggested as an imaging tool to diagnose ASD [11].

The excitation/inhibition (E/I) imbalance observed in different brain regions such as the prefrontal cortex (PFC) has been shown to be critical for social deficits in various ASD mouse models $[1,12,13]$. Along with their genetic heterogeneities, different ASD mouse models exhibit diverse alterations in the excitatory and inhibitory synaptic functions in the neocortex and/or hippocampus (HPC) $[14,15]$. For example, the E/I ratio decreased with reduced $\mathrm{mEPSC}$ amplitude in cortical layer 5 of MECP2 mutant mice or increased mIPSC frequency in layer $2 / 3$ of Neuroligin $3^{\text {R451C }}$ mutant mice $[16,17]$. However, other studies also exhibit increased $\mathrm{E} / \mathrm{I}$ ratio as reduced spontaneous GABAergic inhibitory transmission in BTBR mice or increased mEPSC in the PFC of VPA-exposed mice $[18,19]$. In particular, although alterations in E/I balance have also been reported in contactin-associated proteinlike 2 knockout (Cntnap $2^{-1-}$ ) mice [13, 20-23], the directions of $\mathrm{E} / \mathrm{I}$ ratio alterations are inconsistent depending on the literatures $[13,20,21]$. Since the previous studies were performed using brain slices, we hypothesized that the discrepancy might be due to the ex vivo experimental conditions, such as brain regions and recording conditions. Therefore, we examined the E/I ratio and other metabolites in multiple brain regions of Cntnap $2^{-/-}$mice using the MRS technique in the present study.

\section{Methods}

\section{Mice}

Cntnap 2 knockout $\left(\right.$ Cntnap $\left.2^{--}\right)$mice were a generous gift from Dr. Daniel H. Geschwind (University of California Los Angeles). Twenty-week-old male Cntnap $2^{+/+}$and Cntnap $2^{-l-}$ mice were used for the behavioral tests and 9.4T ${ }^{1} \mathrm{H}$-MRS. The same mice were used for behavioral tests and MRS. Mice were housed with a fixed 12-h light/ dark cycle, and food and water were provided ad libitum.

\section{Social behavior test}

Three-chamber social behavior tests were performed as described previously $[19,24]$. The tests comprised three sessions: (1) habituation, (2) social preference test, and (3) social recognition test. During the habituation session, each subject mouse was introduced to the 3-chamber behavior apparatus and allowed to move freely for $5 \mathrm{~min}$. After habituation, a novel target conspecific was introduced to a wired cup on one side of the chamber, while the other side of the chamber remained empty. Each location for a novel conspecific and empty cup was counterbalanced. After $10 \mathrm{~min}$ of the social preference test, another novel conspecific was introduced to the empty cup for the social recognition test. The subject mouse was allowed to explore conspecifics for another $10 \mathrm{~min}$ during the social recognition test. Behavior sessions were recorded and analyzed manually, while the experimenters were blinded to the genotype of the subject mice. To normalize the variation among subject mice, all the behavior data were represented by the percentage of time spent investigating one side out of the summation of investigation times for both sides. The preference index (PI) was calculated using the following equations: $\mathrm{ET}_{\mathrm{M}}$, exploration time for a mouse; $\mathrm{ET}_{\mathrm{E}}$, exploration time for an empty cup; $\mathrm{ET}_{\mathrm{N}}$, exploration time for a novel mouse; $\mathrm{ET}_{\mathrm{F}}$, exploration time for a familiar mouse).

$$
\begin{gathered}
\text { PIforsocialpreference }=\frac{E T_{M}-E T_{E}}{E T_{M}+E T_{E}} \\
\text { PIforsocialrecognition }=\frac{E T_{N}-E T_{F}}{E T_{N}+E T_{F}}
\end{gathered}
$$

\section{Magnetic resonance spectroscopy (MRS)}

MRS data were acquired on a 9.4T animal MR system (Agilent Technologies, USA) using a volume coil (inner diameter $=72 \mathrm{~mm}$ ) for $\mathrm{RF}$ transmission and a surface coil (phased array 2-channel for mouse brain) for signal reception (Rapid Biomedical GmbH, Rimpar, Germany). T2-weighted scout images were acquired in the axial, coronal, and sagittal planes using a multi-slice turbo spin-echo sequence (repetition time $[\mathrm{TR}]=3500 \mathrm{~ms}$, effective echo time $\left[\mathrm{TE}_{\text {eff }}\right]=30 \mathrm{~ms}$, echo train length $[\mathrm{ETL}]=6$, field of view $[\mathrm{FOV}]=20 \times 20 \mathrm{~mm}^{2}$, matrix size $=128 \times 128$, slice thickness $=0.8 \mathrm{~mm}$, number of averages $(\mathrm{NA})=1$, and scan time $=1 \min 24 \mathrm{~s}$ ). The spectroscopic voxel was positioned in the prefrontal cortex $\left(1.6 \times 1.2 \times 1.5 \mathrm{~mm}^{3}\right)$ and hippocampus $(1.3 \times 1.3 \times 1.9$ $\mathrm{mm}^{3}$ ) (Fig. 1) according to the mouse brain atlas. The static field homogeneity over the voxel was manually adjusted using first- and second-order shimming. Water-suppressed ${ }^{1} \mathrm{H}$ MRS was performed using a pointresolved spectroscopy (PRESS) sequence (TR $=3000 \mathrm{~ms}$, $\mathrm{TE}=15 \mathrm{~ms}, \mathrm{NA}=384$, number of data points $=2048$, spectral bandwidth $=5000 \mathrm{~Hz}$, and scan time $=32 \mathrm{~min}$ ) [25]. An outer volume suppression module was used before the main sequence, which was interleaved with a water suppression module using variable-power RF 
pulses with optimized relaxation delays (VAPOR) [26, 27]. A non-water-suppressed spectrum was also acquired for each voxel $(\mathrm{NA}=8)$.

Metabolite quantification was performed using the LCModel (version 6.3-1L) with the vendor-provided spectral basis set [28]. The absolute concentrations of the following 17 metabolites were estimated: alanine (Ala), aspartate (Asp), creatine (Cr), GABA, glucose (Glc), glutamine $(\mathrm{Gln})$, glutamate $(\mathrm{Glu})$, glutathione $(\mathrm{GSH})$, glycerophosphorylcholine (GPC), lactate (Lac), myo-inositol (Ins), $\mathrm{N}$-acetylaspartate (NAA), N-acetylaspartylglutamate (NAAG), PCr (Phosphocreatine), phosphorylcholine (PCh), scyllo-inositol (Scyllo), and taurine (Tau). Only those results with Cramér-Rao-lower-bounds (CRLB, $\% \mathrm{SD}) \leq 50 \%$ were included in the statistical analysis $[29$, 30].

\section{Statistical analysis}

Mouse social behaviors tested in 3-chamber test were compared using two-way ANOVA with Sidak's multiple comparisons test, while the preference indices were analyzed using a two-tailed unpaired t-test with Welch's correction. Metabolite levels and ratios were also examined using a two-tailed unpaired t-test with Welch's correction. The correlation coefficient $(r)$ between mice social behaviors and levels or ratios of metabolites was calculated with linear regression using the Pearson correlation method. To compute the significance of correlation, we generated empirical null distributions of correlation coefficients by randomly permuting the samples 5000 times. Based on empirical null distributions, we computed adjusted $\mathrm{p}$-values for the Pearson correlation (Pr). Metabolites with $\operatorname{Pr}<0.05$ were selected as key metabolites. Metabolites with $\operatorname{Pr}<0.05$ and $\operatorname{Pr}<0.025$ are marked in the figure described as $95 \%$ or $97.5 \%$ quantiles, respectively. Differential expression of key metabolites between Cntnap2 ${ }^{+/+}$and Cntnap2 $2^{-1-}$ mice were tested using Student's t-test using customized MATLAB code. For the key metabolites, simple logistic regression classification was carried out to determine the concentration with the highest discriminant power between the two groups. Given the small sample size, all the samples were used to train the model. Specificity, sensitivity, and optimal concentration criteria for discriminating the two groups were assessed using receiver operating characteristic (ROC) curves in the binormal approach, represented by corresponding area under the curve (AUC) values with 95\% confidence level. All statistical analyses except the permutation test were performed using the GraphPad Prism 7.00. Mouse social behaviors and preference index are shown as mean \pm standard error of the mean (S.E.M.), while the amounts of metabolites or the ratio of different metabolites are shown as mean \pm standard deviation (S.D.).

\section{Results \\ Cntnap $^{-1-}$ mice show impaired social behaviors}

Mutations in the Cntnap2 gene are strongly associated with ASD [31]. Cntnap $2^{-1-}$ mice show core ASD symptoms, such as reduced vocalization/social interaction and

\footnotetext{
(See figure on next page.)

Fig. 1 Cntnap2-null mice show deficits in social behaviors. a Schematic diagram for three-chamber social behavior tests. Social preference test was followed by social recognition test. $\mathbf{b}$ Schematic diagram for $9.4 \mathrm{~T}^{1} \mathrm{H}$-magnetic resonance spectroscopy (MRS) for mouse. $\mathbf{c}$ In the social

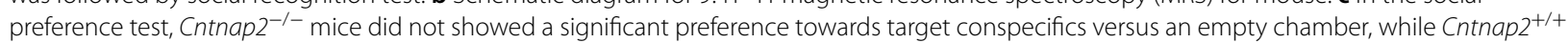
mice showed a significant preference towards target conspecifics. Two-way ANOVA: Cntnap2 ${ }^{+/+}, n=9$ mice, Cntnap2 ${ }^{-/-}, n=11$ mice, interaction between genotype $\times$ target, $F_{1,34}=97.71,{ }^{* * * *} P<0.0001$; Sidak's multiple comparisons test: Cntnap2 ${ }^{+/+}$, mouse versus object $t_{34}=13.49$, ${ }^{* * * *} P<0.0001$; Cntnap2 $2^{-/}$, mouse versus object $t_{34}=0.5863, P=0.5863$. n.s., not significant. $\mathbf{d}$ Deletion of Cntnap2 gene significantly affect the social preference index in Cntnap2 ${ }^{-1-}$ mice. Two-tailed unpaired t-test with Welch's correction, ${ }^{* * *} P<0.0001$. e In the social recognition test, Cntnap2 $2^{-1-}$ mice did not showed a significant preference towards novel conspecifics versus familiar conspecifics, while Cntnap2 $2^{+/+}$mice showed a significant preference towards novel conspecifics. Two-way ANOVA: Cntnap2 ${ }^{+/+}, \mathrm{n}=9$ mice, Cntnap $2^{-/-}, \mathrm{n}=11$ mice, interaction between genotype $\times$ social target, $F_{1,34}=2.882, P=0.0987$; Sidak's multiple comparisons test: Cntnap2 ${ }^{+/+}$, novel mouse versus familiar mouse $t_{34}=3.954$, ${ }^{* * *} P=0.0007$; Cntnap2 $2^{-/}$, novel mouse versus familiar mouse $t_{34}=2.02, P=0.0999$. n.S., not significant. $\mathbf{f}$ Deletion of Cntnap2 gene did not affect the social preference index for novel mouse in $\mathrm{Cntnap2}^{-1-}$ mice. Two-tailed unpaired t-test with Welch's correction, $P=0.3010$. $\mathbf{g}$ Example voxel images targeting prefrontal cortex and hippocampus of mouse as coronal, axial and sagittal planes. $\mathbf{h}$ Example image of $9.4 \mathrm{~T}^{1} \mathrm{H}-\mathrm{MR}$ spectra shown as a black trace. Red trace shows the result of $\mathrm{LC}$ model fit. (i) Comparison of metabolite concentrations monitored by $9.4 \mathrm{~T}^{1} \mathrm{H}-\mathrm{MRS}$ in the prefrontal cortex. Metabolites which number of individuals with $C R L B \leq 50 \%$ is greater than 5 were included for statistical analysis (Ala: Cntnap2 ${ }^{+/+}, n=8$; Cntnap2 $^{-/-}, \mathrm{n}=10 ;$ Asp: Cntnap2 $2^{+/+}, \mathrm{n}=9 ;$ Cntnap2 $^{-/-}, \mathrm{n}=11 ;$ Cr: Cntnap2 $^{+/+}, \mathrm{n}=5 ;$ Cntnap2 $^{-/-}, \mathrm{n}=10 ; \mathrm{PCr}:$ Cntnap2 $^{+/+}, \mathrm{n}=9 ;$ Cntnap2 $^{-/-}$, $\mathrm{n}=11$; GABA: Cntnap2 $2^{+/+}, \mathrm{n}=8 ;$ Cntnap2 $^{-/-}, \mathrm{n}=11 ; \mathrm{Gln}$ : Cntnap2 $2^{+/+}, \mathrm{n}=9 ;$ Cntnap2 $^{-/-}, \mathrm{n}=11 ; \mathrm{Glu}:$ Cntnap2 $^{+/+}, \mathrm{n}=9 ;$ Cntnap2 $^{-/-}, \mathrm{n}=11 ; \mathrm{GPC}$ Cntnap2 $^{+/+}, \mathrm{n}=8 ;$ Cntnap2 $^{-/-}, \mathrm{n}=9 ; \mathrm{PCh}:$ Cntnap2 $^{+/+}, \mathrm{n}=7 ;$ Cntnap2 $^{-/-}, \mathrm{n}=10 ; \mathrm{GSH}:$ Cntnap2 $^{+/+}, \mathrm{n}=9 ;$ Cntnap2 $^{-/-}, \mathrm{n}=11 ;$ Ins: Cntnap2 ${ }^{+/+}$, $\mathrm{n}=9 ;$ Cntnap2 $^{-/-}, \mathrm{n}=11$; NAA: Cntnap2 ${ }^{+/+}, \mathrm{n}=9 ;$ Cntnap2 $^{-/-}, \mathrm{n}=11 ;$ Tau: Cntnap2 $2^{+/+}, \mathrm{n}=9 ;$ Cntnap2 $\left.^{-/-}, \mathrm{n}=11\right)$. $\mathbf{j}$ Comparison of metabolite concentrations monitored by $9.4 \mathrm{~T}^{1} \mathrm{H}$-MRS in the hippocampus. Metabolites which number of individuals with CRLB $\leq 50 \%$ is greater than 5 were included for statistical analysis (Ala: Cntnap2 $2^{+/+}, \mathrm{n}=9 ;$ Cntnap2 $^{-/-}, \mathrm{n}=10 ;$ Asp: Cntnap2 $^{+/+}, \mathrm{n}=9 ;$ Cntnap2 $^{-/-}, \mathrm{n}=11 ; \mathrm{Cr}_{\mathrm{C}}$ Cntnap2 $2^{+/+}, \mathrm{n}=9$; Cntnap2 $^{-/-}, \mathrm{n}=10 ; \mathrm{PCr}:$ Cntnap2 $^{+/+}, \mathrm{n}=9 ;$ Cntnap2 $^{-/-}, \mathrm{n}=11 ; \mathrm{GABA}^{-}$Cntnap2 $2^{+/+}, \mathrm{n}=9 ;$ Cntnap2 $^{-/-}, \mathrm{n}=11 ;$ Gln: Cntnap2 $^{+/+}, \mathrm{n}=9 ;$ Cntnap2 $^{-/-}$, $\mathrm{n}=11$; Glu: Cntnap2 ${ }^{+/+}, \mathrm{n}=9 ;$ Cntnap2 $^{-/-}, \mathrm{n}=11 ; \mathrm{PCh}_{\text {Cntnap2 }}^{+/+}, \mathrm{n}=8 ;$ Cntnap2 $^{-/-}, \mathrm{n}=9 ; \mathrm{GSH}:$ Cntnap2 $^{+/+}, \mathrm{n}=9 ;$ Cntnap2 $^{-/-}, \mathrm{n}=11 ;$ Ins: Cntnap2 $^{+/+}, \mathrm{n}=9 ;$ Cntnap2 $^{-/-}, \mathrm{n}=11 ;$ NAA: Cntnap2 $^{+/+}, \mathrm{n}=9 ;$ Cntnap2 $^{-/-}, \mathrm{n}=11 ;$ NAAG: Cntnap2 $^{+/+}, \mathrm{n}=8 ;$ Cntnap2 $^{-/-}, \mathrm{n}=9 ;$ Tau: $^{\text {Cntnap2 }}{ }^{+/+}$, $\mathrm{n}=9 ;$ Cntnap2 $\left.^{-/-}, \mathrm{n}=11\right)$
} 
a

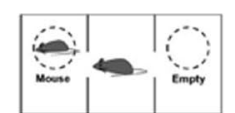

Social preference

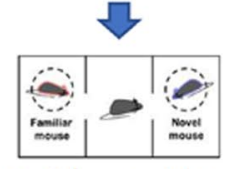

Social recognition

3-chamber test

b

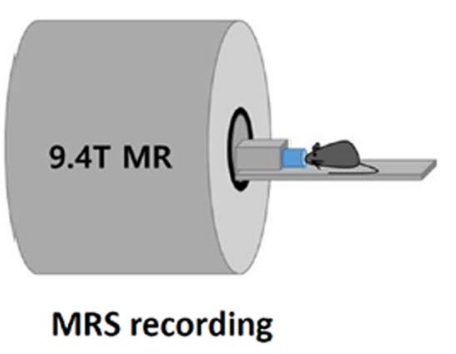

g

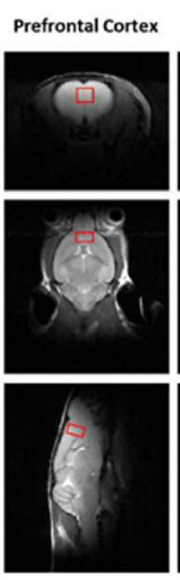

i

Prefrontal cortex

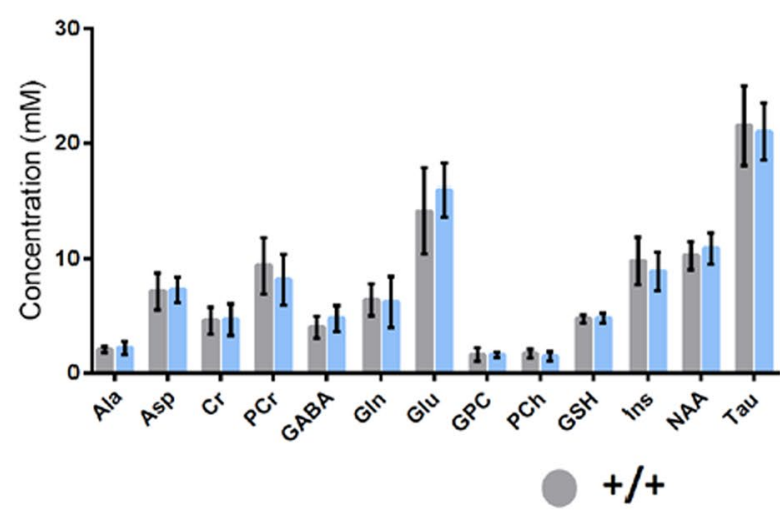

Fig. 1 (See legend on previous page.)
C

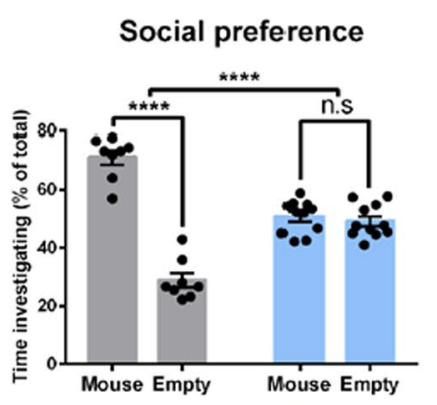

e

$+1+$

$-l-$

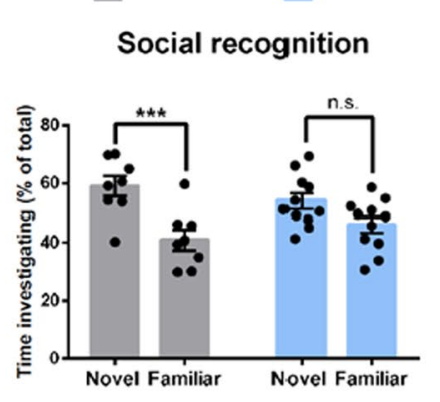

d



h

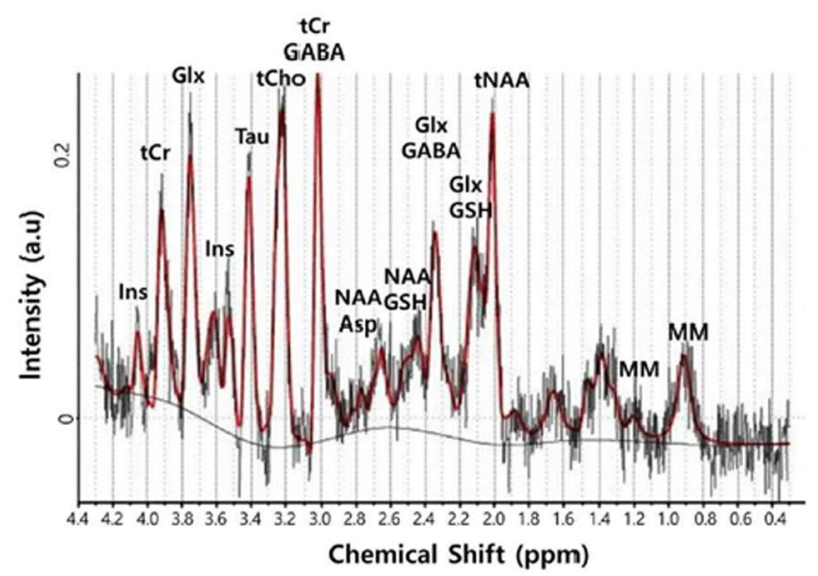

Hippocampus

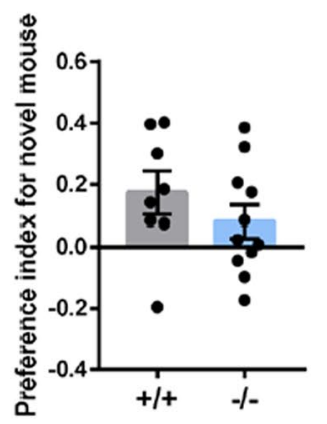

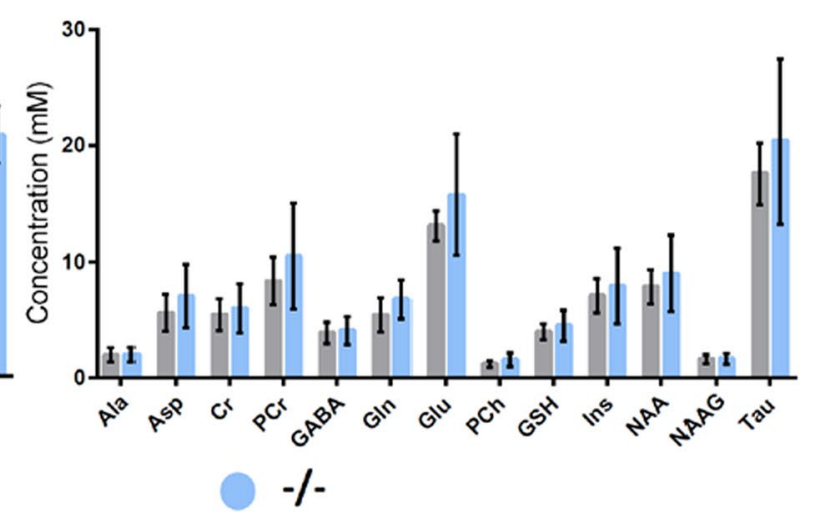


increased repetitive behaviors [32]. To confirm the previously reported social behavior deficits in Cntnap $2^{-1-}$ mice, we first tested Cntnap $2^{-1-}$ mice in the 3-chamber social behavior test (Fig. 1a). In the social preference test, Cntnap $2^{-1-}$ mice showed a comparable level of investigation time towards target conspecifics compared to that for an empty chamber, while Cntnap $2^{+/+}$mice showed a significant preference for target conspecifics (Fig. 1c). Meanwhile, Cntnap $2^{-1-}$ mice showed a significantly lower preference index for novel mice compared to Cnt$n a p 2^{+/+}$mice (Fig. 1d). In addition, Cntnap $2^{-/-}$mice did not exhibit a significant preference for novel conspecifics during the social recognition test, whereas Cntnap $2^{+/+}$ mice showed a significant preference for novel conspecifics compared to that for familiar conspecifics (Fig. 1e). However, the preference index for novel mice shown by Cntnap $2^{-1-}$ mice was not significantly lower than that of Cntnap $2^{+/+}$mice (Fig. 1f). These results suggest that Cntnap $2^{-1-}$ mice have a severely impaired social preference, and mildly impaired social recognition. We used Cntnap $2^{-1-}$ mice with a confirmed social preference deficit for $9.4 \mathrm{~T}{ }^{1} \mathrm{H}$-MRS recording, 2 weeks after the 3chamber social behavior test (Fig. 1b). Metabolites from the prefrontal cortex and hippocampus, which are known to govern social behavior, were examined by $9.4 \mathrm{~T}{ }^{1} \mathrm{H}$ MRS [12, 33, 34] (Fig. 1g and h). In the raw data set, we found that all the metabolites analyzed showed comparable concentrations between Cntnap $2^{-/-}$and Cntnap $2^{+/+}$ mice (Fig. 1i and j, Additional file 1: Tables S1 and S2). We analyze the MRS data more closely in the following section.

\section{Several brain metabolites level or their ratio show high correlation with mice sociability}

Using the metabolites whose concentrations were measured by $9.4 \mathrm{~T}{ }^{1} \mathrm{H}$-MRS, we analyzed the relationship between metabolite levels and mice sociability. To examine the correlation, mice social preference and social recognition index were compared with each metabolite in the prefrontal cortex and hippocampus (Fig. 2a, see Additional file 1: Table S1 and S2). There were several candidate metabolites in the prefrontal cortex that showed a significant correlation with social preference (Fig. 2a). Notably, there were positive correlations between social preference and the Glu/GABA ratio and $\mathrm{tCr}$ level in the prefrontal cortex, even though the levels of metabolite substrates were comparable between groups (Figs. 1i, $2 \mathrm{~b}$, and $\mathrm{c}$ ). In addition, the social preference index was negatively correlated with the prefrontal $\mathrm{NAA} / \mathrm{tCr}$ ratio (Fig. 2d). However, there was no metabolite in the prefrontal cortex which showed a significant correlation with social recognition (Additional file 1: Fig. S1a). Furthermore, no metabolite in the hippocampus showed a significant correlation with social preference (Additional file 1: Fig. S1b). Consistent with the notion that hippocampal function is crucial for social recognition [33, 34], we found that the $\mathrm{PCr} / \mathrm{tCr}$ ratio in the hippocampus was negatively correlated with the social recognition ability of mice, but not with the social preference (Fig. 2e, $\mathrm{f}$ and Additional file 1: Fig. S2e). Neither the Glu/GABA and NAA/tCr ratios nor $\mathrm{tCr}$ levels in the prefrontal cortex showed a significant correlation with social recognition, suggesting that these metabolites in the prefrontal cortex are selectively correlated with social preference, but not social recognition in mice (Additional file 1: Fig. S2a-c).

\section{Cntnap2 $^{-1-}$ mice exhibit abnormal metabolic regulation in the prefrontal cortex or in the hippocampus}

Based on their relationships with social behaviors, metabolites exhibiting significant correlation with sociability, as measured by the social preference index, were compared between genotypes. Interestingly, as its alteration is commonly reported in multiple ASD mouse models including Cntnap $2^{-/-}$mice, the Glu/GABA ratio in the prefrontal cortex was significantly lower in Cntnap $2^{-1-}$ mice than in Cntnap2 $2^{+/+}$mice [14, 32, 35] (Fig. 3a). Cntnap2 $2^{-/-}$ mice also showed significantly lower level of $\mathrm{tCr}$ and higher NAA/tCr ratio in the prefrontal cortex compared to Cntnap $2^{-1-}$ mice (Fig. 3b and c). In addition, these metabolic changes between genotypes were not observed in the hippocampus (Fig. $3 \mathrm{f}-\mathrm{h}$ ). In addition, although $\mathrm{PCr} / \mathrm{tCr}$ in the hippocampus showed a significant correlation with the social recognition ability of mice, Cnt$n a p 2^{-/-}$and Cntnap $2^{+/+}$mice showed comparable levels of the $\mathrm{PCr} / \mathrm{tCr}$ ratio in the hippocampus (Figs. $2 \mathrm{f}$ and $3 \mathrm{i}$ ). The $\mathrm{PCr} / \mathrm{tCr}$ ratio was also comparable between genotypes in the prefrontal cortex (Fig. 3d). In addition, the levels of Glx, which include glutamate and glutamine, were not significantly different between Cntnap $2^{-/-}$and Cntnap $2^{+/+}$mice in the prefrontal cortex but were significantly higher in the hippocampus of Cntnap $2^{-1-}$ mice than in Cntnap2 $2^{+/+}$mice (Fig. 3e and j). However, there was no correlation between Glx levels and mouse behavior either in the prefrontal cortex or hippocampus (Additional file 1: Fig. S2d and f), suggesting that Glx might not be an ideal biomarker for social behavior.

\section{Discrimination power of prefrontal Glu/GABA ratio, NAA/ $\mathrm{tCr}$ ratio or $\mathrm{tCr}$ level on Cntnap2 genotype}

To examine the discriminant power of the prefrontal metabolites, we generated a simple logistic regression model and tested it using receiver operating characteristic (ROC) curves. The prefrontal Glu/GABA ratio had an AUC value of 0.838 with a sensitivity of $77.78 \%$ and specificity of $90.91 \%$, suggesting that the prefrontal Glu/GABA 



C

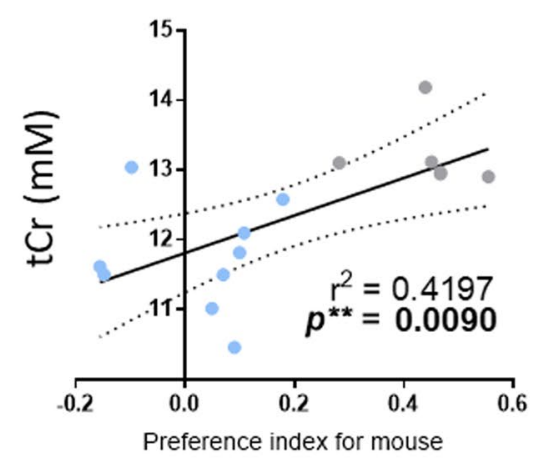

e

\section{Correlation between social recognition} and metabolites in the hippocampus

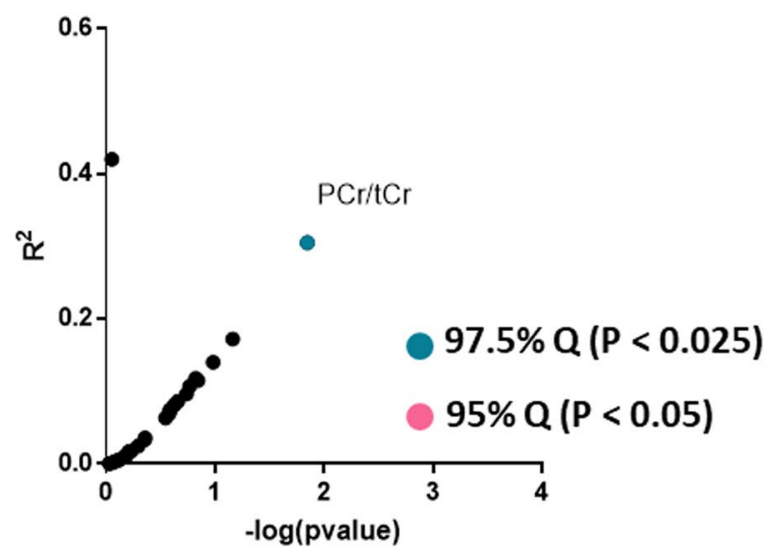

f
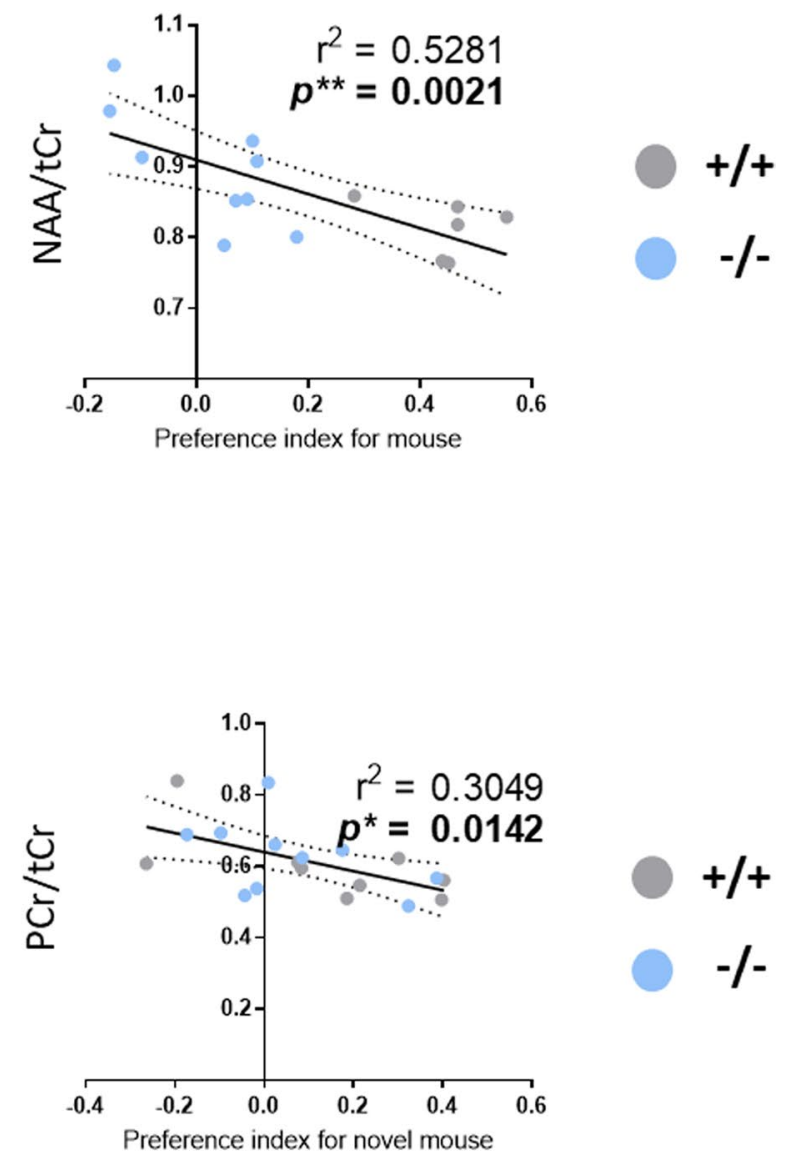

Fig. 2 Linear regression analyses between metabolites and rodent social behavior. a Volcano plot exhibiting a correlation between social preference and the metabolites in the prefrontal cortex. Metabolites with a significant correlation was colored as pink or cyan depending on the quantile of actual Pearson's coefficient from the permutated values, $95 \%(P<0.05)$ or $97.5 \%(P<0.025)$ respectively. $\mathbf{b}$ - $\mathbf{d}$ Correlation between mice social preference index and Glu/GABA, tCr or NAA/tCr. Pearson Correlation Coefficients was used to evaluate linear correlations. Each linear regression line is shown with 95\% confidence bands (two dotted lines). The degree of significant correlation $(P)$ and goodness of fit $\left(r^{2}\right)$ were written in each figure. e Volcano plot exhibiting a correlation between social recognition and metabolites in hippocampus. Metabolites with a significant correlation was colored as pink or cyan depending on the quantile of actual Pearson's coefficient from the permutated values, $95 \%$ or $97.5 \%$ respectively. $\mathbf{f}$ Correlation between mice social preference index for novel mouse and $\mathrm{PCr} / \mathrm{tCr}$ 


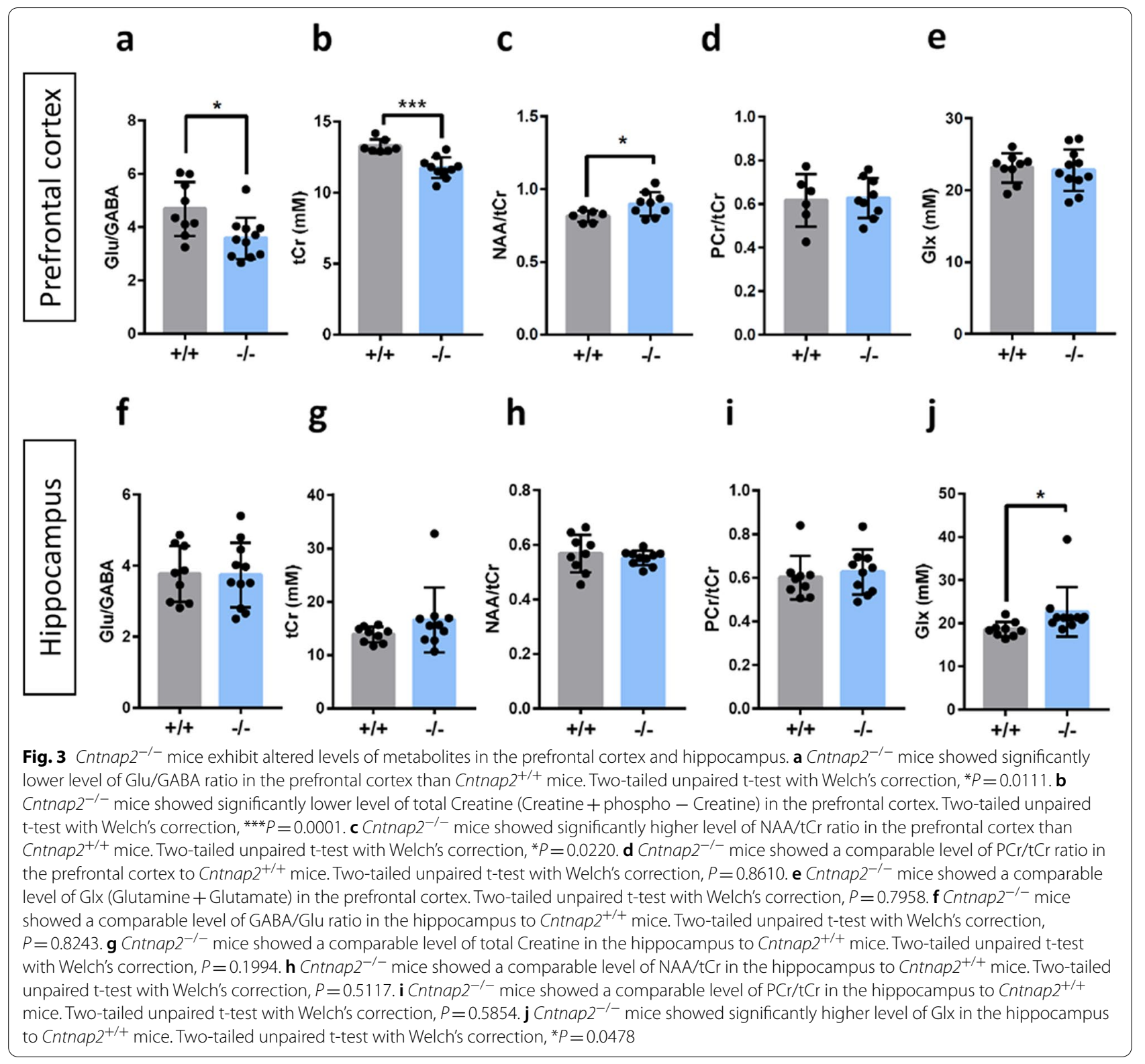

ratio can be used to properly distinguish Cntnap $2^{+/+}$and Cntnap $2^{-1-}$ mice (Fig. 4a and d). Consistent with its comparable level between groups, such a high prediction was not shown using glutamate only (Additional file 1: Fig. S3a and d). Along with the significant difference between groups, tCr level and NAA/tCr ratio also showed significantly accurate predictive scores (Fig. $4 \mathrm{~b}-\mathrm{d}$ ). Such metabolic prediction was not precise enough to predict the mouse genotype when using either $\mathrm{PCr}$ or NAA alone (Additional file 1: Fig. S3b-d).

\section{Discussion}

Neuroimaging biomarkers such as functional connectivity and brain volume have been introduced as ASD biomarkers [36, 37]. In several human cases with Cntnap 2 mutations, focal malformation of brain regions or reduced long-range connectivity have been reported $[38,39]$. Even though MRS has also been used to reveal abnormal levels of metabolites not only in ASD rodent models, but also in humans with ASD [40, 41], patients with Cntnap2 mutations were scarcely reported for 

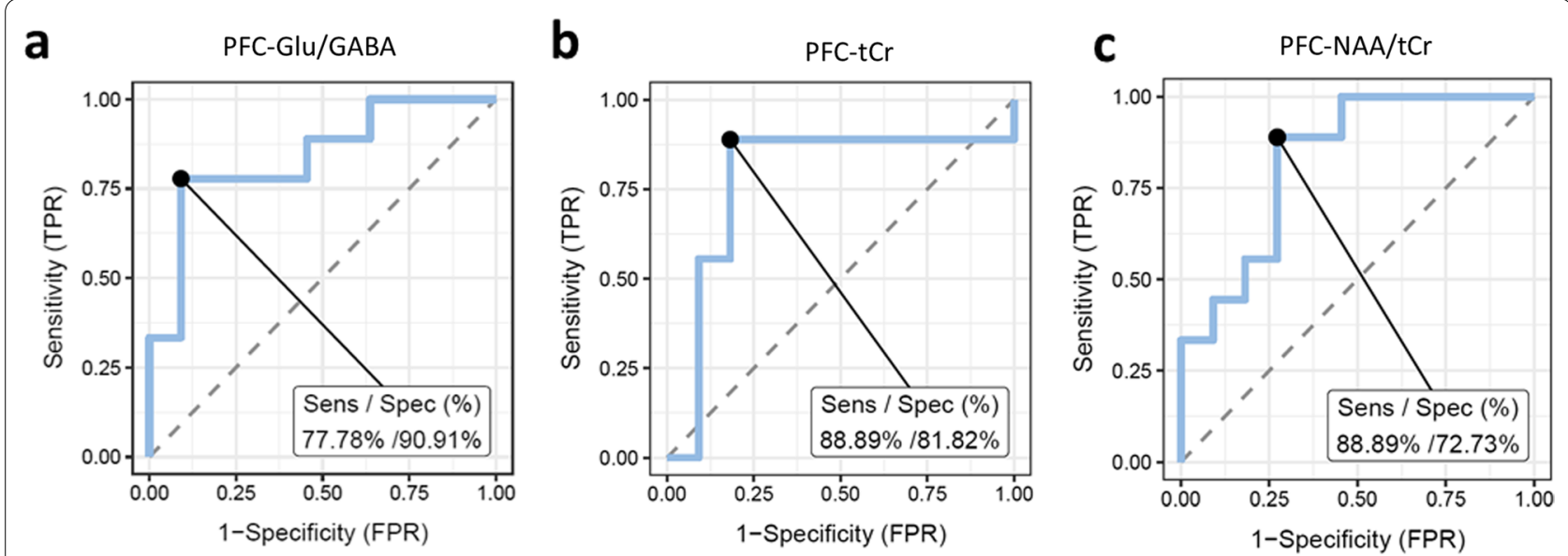

\begin{tabular}{|c|c|c|c|}
\hline \multicolumn{1}{|c|}{ Glu/GABA } & tCr & NAA/tCr \\
\hline & 0.838 & 0.778 & 0.828 \\
\hline AUC & $0.651-1$ & $0.564-0.992$ & $0.636-1$ \\
\hline (95\% Cl) & 0.687 & 0.707 & 0.616 \\
\hline Youden Index & -0.14 & 0.045 & -0.251 \\
\hline Cut-off(log-odds) & & & \\
\hline
\end{tabular}

Fig. 4 Prefrontal $\mathrm{Glu} / \mathrm{GABA}, \mathrm{tCr}$, and $\mathrm{NAA} / \mathrm{tCr}$ precisely predict the genotype. a-c $\mathrm{ROC}$ curve analyses of metabolites showing a significant correlation with social preference. $\mathbf{d}$ Information table with detail description for each ROC analysis

their brain metabolic changes [42]. There were limited studies reported altered metabolites in patients with Cntnap 2 mutant variants [43, 44]. In the present study, we found strong correlations between the social preference index and Glu/GABA, NAA/tCr ratio, and tCr level in the prefrontal cortex of Cntnap $2^{-1-}$ mice using in vivo MRS technique that suggests using these metabolites as biomarkers for social preference phenotypes in ASD. Since ASD exhibits high comorbidity with other neurodevelopmental disorders such as intellectual disability, epilepsy, and attention deficit hyperactivity disorder, finding biomarkers for a specific phenotype such as social preference seems to be more reliable, rather than finding biomarkers for individual disorders [45-47].

$\mathrm{E} / \mathrm{I}$ imbalance has been suggested as one of the mechanisms underlying behavioral phenotypes associated with ASD [14, 15]. Hence, detecting changes in the $\mathrm{E} / \mathrm{I}$ balance has been suggested as a mechanism-based diagnostic index for ASD [48]. However, it is worth noting that the $\mathrm{E} / \mathrm{I}$ imbalance has been shown to be brain region-dependent both in humans and mice [35, 49-52].
For example, Horder et al. (2018) reported that Glu concentration was reduced in the striatum, but not in the prefrontal cortex, in humans diagnosed with ASD using MRS [35]. An MRS study using $N f 1^{+/-}$mice, a mouse model of neurofibromatosis type 1 , showed increased GABA/Glu ratios in the prefrontal cortex and striatum, but not in the hippocampus [53]. We also found that the Glu/GABA ratio is significantly changed only in the prefrontal cortex in Cntnap $2^{-/-}$mice, highlighting that examining the $E / I$ balance in a region-specific manner is critical for investigating cellular mechanisms or developing diagnostic biomarkers for ASD or other neurodevelopmental disorders. Such a region-specific E/I imbalance in Cntnap $2^{-1-}$ mice may be derived not only from the abundancy of Cntnap 2 differ by brain regions, but also from the expression of downstream molecules of Cntnap2 [32, 54]. Mechanisms underlying brain region-specific E/I imbalance associated with ASD would be an important subject for further investigation.

Even though E/I imbalance has been suggested as a common mechanism for ASD and other neurodevelopmental 
disorders, the direction of change depends on the conditions. $[17,19]$. More interestingly, there are inconsistent results regarding the direction of the $\mathrm{E} / \mathrm{I}$ ratio changes in Cntnap $2^{-1-}$ mice $[13,21]$. We found that the E/I ratio measured by the Glu/GABA ratio using MRS was significantly decreased in the prefrontal cortex, but not in the hippocampus of Cntnap $2^{-1-}$ mice. This result is consistent with previous ex vivo studies that show a reduction in excitatory synaptic transmission in the prefrontal cortex or in the visual cortex of adult Cntnap $2^{-/-}$mice $[20,55]$. In contrast, other studies have suggested reduced inhibition in the cortex of Cntnap $2^{-/-}$mice, suggesting that Cntnap2 deletion may increase the E/I ratio $[13,32,56]$. These seemingly inconsistent results may stem from subtle differences in experimental conditions, such as the age of the animals. It has been suggested that a discrepancy in $\mathrm{E} / \mathrm{I}$ ratio can occur at different developmental stages within a single mouse subject $[56,57]$. In this study, due to burdensome hours under anesthesia for adolescent mice, we used 20-week-old adult mice for MRS. Longitudinal MRS study in the same mice subjects or human with ASD would provide more information on the dynamic changes of the $\mathrm{E} / \mathrm{I}$ ratio in multiple brain regions.

It should be noted that the Glu/GABA ratio imbalance assessed by metabolites in this study and the synaptic E/I imbalance reported in previous studies may have different meanings. The decreased Glu/GABA ratio may not be directly translated into the decreased synaptic E/I imbalance since the synaptic E/I balance can be regulated not only by the concentration of neurotransmitters, but also by homeostatic or metaplastic mechanisms such as adjusting the number or activity of postsynaptic receptors $[58,59]$. It would be interesting to test whether the manipulation of Glu or GABA actions using their receptor agonists or antagonists can affect mice behavior or cognitive ability in Cntnap $2^{-1-}$ mice as shown in other mouse models of neurodevelopmental disorders [60-62].

It is known that $~ 5 \%$ of ASD cases are reported for their potential to attribute inborn errors of metabolism [63, 64]. Children with creatine deficiency syndrome show autistic-like features and behaviors, such as developmental delay and intellectual disability [65-67]. Reduction of $\mathrm{tCr}$ in children with ASD was also observed not only in the grey matter but also in the white matter with agedependent variations $[68,69]$. Similarly, creatine transporter (Slc6a8) knockout mice exhibited significantly low creatine levels within the brain, resulting in behavioral phenotypes such as hyperactivity and impaired learning and memory, as shown in other ASD mouse models [67]. Together with our finding of decreased total creatine levels in the prefrontal cortex of Cntnap $2^{-1-}$ mice, these results suggest that total creatine is also a promising biomarker for social preference deficits in ASD.
Reduced NAA/tCr ratios have been reported in diverse cases, such as patients with Alzheimer's disease, euthymic bipolar disorder, or men with recreational cannabis usage [70-72]. Interestingly, increased $\mathrm{NAA} / \mathrm{tCr}$ levels have rarely been reported. Recently, NAA has been commonly discussed as a metabolite that indicates neural dysfunction by various factors, including neuroinflammation [73]. Although further confirmation is essential, based on its unique feature of elevated ratio in this ASD mouse model, high correlation with mouse behavior, and significant alteration by genotype, the NAA/tCr ratio can be suggested as one of the potential biomarkers like the other two metabolic alterations discussed above.

We tested whether the metabolites could be used to predict the Cntnap 2 genotypes by using ROC curve analysis. We found that the prefrontal Glu/GABA ratio, $\mathrm{NAA} / \mathrm{tCr}$ ratio or $\mathrm{tCr}$ level have strong discrimination power on the genotype, suggesting that these metabolites might be useful imaging biomarkers for predicting social deficits associated with ASD. However, using metabolites to diagnose ASD needs to be further confirmed using several other types of biomarkers because dysregulated metabolites could be an epiphenomenon of ASD, which requires additional studies to confirm their correlations [74]. It should also be noted that our results are derived from one mutant mouse line and the relatively small number of animals were used in this study.

\section{Conclusions}

In this study, by using ${ }^{1} \mathrm{H}$-MRS and behavioral tests, we found that the Glu/GABA ratio, NAA/tCr ratio, and $\mathrm{tCr}$ level in the prefrontal cortex are significantly correlated with the social preference index of mice. These metabolites were significantly different in Cntnap $2^{-/-}$mice compared to those in wild-type mice. Moreover, these metabolites showed high specificity and selectivity in discriminating Cntnap2 mutant from wild-type mice. Our study suggests that the accuracy of diagnosing ASD might be substantially improved by using multimodal approaches such as the non-invasive MRS technique.

\footnotetext{
Abbreviations

Ala: Alanine; ASD: Autism spectrum disorder; Asp: Aspartate; Cr: Creatine; GABA: Y-Aminobutyric acid; Gln: Glutamine; Glu: Glutamate; GPC: Glycerylphosphorylcholine; GSH: Glutathione; Glx: Glutamate + glutamine; HPC: Hippocampus; Ins: Myo-inositol; mEPSC: Miniature excitatory postsynaptic currents; MM: Macromolecule; MRI: Magnetic resonance imaging; MRS: Magnetic resonance spectroscopy; NAA: N-acetylaspartate; NAAG: N-acetylaspartylglutamate; PCr: Phosphocreatine; PCh: Phosphorylcholine; PFC: Prefrontal cortex; ROC: Receiver operating characteristics; scyllo: Scyllo-inositol; tau: Taurine; tCr: Total creatine; tCho: PCh + GPC; tNAA: NAA + NAAG.
} 


\section{Supplementary Information}

The online version contains supplementary material available at https://doi. org/10.1186/s13041-022-00904-z.

Additional file 1. Table S1. Total metabolites in the prefrontal cortex. Table S2. Total metabolites in the hippocampus. Supplementary figure S1. Volcano plot showing significant Pearson's correlation between metabolites and rodent sociability. Supplementary figure S2. Other linear regression analyses between metabolites and rodent sociability. Supplementary figure S3. ROC analyses between metabolites and rodent genotype.

\section{Acknowledgements}

Not applicable.

\section{Authors' contributions}

GP, HJK, and Y-SL designed the experiments and wrote the manuscript. GP, IOK, KCL, and HJK performed 9.4T ${ }^{1} \mathrm{H}$-MRS imaging and analyzed the data. SJJ performed all behavioral experiments and analyses. JHP, MSK, and CYS analyzed the data and edited the manuscript. All authors read and approved the final manuscript.

\section{Funding}

This work was supported by the National Research Foundation of Korea (Grant numbers NRF-2017M3C7A1026959 and NRF-2019R1A2C1084232 to Y.-S.L. and NRF-2018H1A2A1061381 to G.P.); and Ministry of Science and ICT (Grant number 50536-2021 to K.C.L.)

\section{Availability of data and materials}

All data generated or analyzed in this study are included in this published article.

\section{Declarations}

Ethics approval and consent to participate

This study was approved by the Institutional Animal care and Use Committee of Konkuk University (approval number KU20193) and Seoul National University (approval number SNU 171220-2-5).

\section{Consent for publication}

Not applicable.

\section{Competing interests}

The authors declare that they have no competing interests.

\begin{abstract}
Author details
'Department of Biomedical Sciences, BK21 Four Biomedical Science Program, Seoul National University College of Medicine, Seoul, Republic of Korea. ${ }^{2}$ Department of Physiology, Seoul National University College of Medicine, Seoul, Republic of Korea. ${ }^{3}$ School of Medicine and Center for Neuroscience Research, Konkuk University, Seoul, Republic of Korea. ${ }^{4}$ Division of Applied RI, Korea Institute Radiological and Medical Sciences, Seoul, Republic of Korea. ${ }^{5}$ Department of New Biology, Daegu Gyeongbuk Institute of Science and Technology (DGIST), Daegu, Republic of Korea. ${ }^{6}$ Department of Medical Sciences, Seoul National University College of Medicine, Seoul, Republic of Korea. ${ }^{7}$ Neuroscience Research Institute, Seoul National University College of Medicine, Seoul, Republic of Korea. ${ }^{8}$ Wide River Institute of Immunology, Seoul National University, Hongcheon, Republic of Korea.
\end{abstract}

Received: 11 January 2022 Accepted: 8 February 2022

Published online: 19 February 2022

\section{References}

1. Diagnostic and statistical manual of mental disorders: DSM-5 ${ }^{\mathrm{TM}}$, 5 th ed. Arlington, VA, US: American Psychiatric Publishing, Inc.; 2013. xliv, 947xliv, p.
2. Hyman SL, Levy SE, Myers SM, Council On Children With Disabilities SOD, Behavioral P, Kuo DZ, et al. Identification, evaluation, and management of children with autism spectrum disorder. Pediatrics. 2020;145(1).

3. Shi B, Wu W, Dai M, Zeng J, Luo J, Cai L, et al. Cognitive, language, and behavioral outcomes in children with autism spectrum disorders exposed to early comprehensive treatment models: a meta-analysis and meta-regression. Front Psychiatry. 2021;12(1234).

4. Vietze P, Lax LE. Early intervention ABA for toddlers with ASD: effect of age and amount. Curr Psychol. 2020;39(4):1234-44.

5. Jones W, Klin A. Attention to eyes is present but in decline in 2-6-monthold infants later diagnosed with autism. Nature. 2013;504(7480):427-31.

6. Muller CL, Anacker AMJ, Veenstra-VanderWeele J. The serotonin system in autism spectrum disorder: from biomarker to animal models. Neuroscience. 2016;321:24-41.

7. Mulder EJ, Anderson GM, Kema IP, de Bildt A, van Lang NDJ, den Boer JA, et al. Platelet serotonin levels in pervasive developmental disorders and mental retardation: diagnostic group differences, within-group distribution, and behavioral correlates. J Am Acad Child Adolesc Psychiatry. 2004;43(4):491-9.

8. Plitt M, Barnes KA, Martin A. Functional connectivity classification of autism identifies highly predictive brain features but falls short of biomarker standards. Neurolmage Clin. 2015;7:359-66.

9. Bosl WJ, Tager-Flusberg H, Nelson CA. EEG analytics for early detection of autism spectrum disorder: a data-driven approach. Sci Rep. 2018;8(1):6828.

10. Vaccarino FM, Smith KM. Increased brain size in autism-what it will take to solve a mystery. Biol Psychiat. 2009;66(4):313-5.

11. Mori K, Toda Y, Ito H, Mori T, Mori K, Goji A, et al. Neuroimaging in autism spectrum disorders: $1 \mathrm{H}-\mathrm{MRS}$ and NIRS study. J Med Invest. 2015:62(1-2):29-36.

12. Yizhar O, Fenno LE, Prigge M, Schneider F, Davidson TJ, O'Shea DJ, et al. Neocortical excitation/inhibition balance in information processing and social dysfunction. Nature. 2011;477(7363):171-8.

13. Selimbeyoglu A, Kim CK, Inoue M, Lee SY, Hong ASO, Kauvar I, et al. Modulation of prefrontal cortex excitation/inhibition balance rescues social behavior in CNTNAP2-deficient mice. Sci Transl Med. 2017;9(401):eaah6733.

14. Lee E, Lee J, Kim E. Excitation/inhibition imbalance in animal models of autism spectrum disorders. Biol Psychiat. 2017;81(10):838-47.

15. Kim KC, Gonzales EL, Lzaro Ma T, Choi CS, Bahn GH, Yoo HJ, et al. Clinical and neurobiological relevance of current animal models of autism spectrum disorders. Biomol Therapeutics. 2016;24(3):207-43.

16. Dani VS, Chang Q, Maffei A, Turrigiano GG, Jaenisch R, Nelson SB. Reduced cortical activity due to a shift in the balance between excitation and inhibition in a mouse model of Rett Syndrome. Proc Natl Acad Sci USA. 2005;102(35):12560.

17. Tabuchi K, Blundell J, Etherton Mark R, Hammer Robert E, Liu X, Powell Craig $M$, et al. A neuroligin-3 mutation implicated in autism increases inhibitory synaptic transmission in mice. Science. 2007;318(5847):71-6.

18. Han S, Tai C, Jones Christina J, Scheuer T, Catterall WA. Enhancement of inhibitory neurotransmission by GABAA receptors having a2,3-subunits ameliorates behavioral deficits in a mouse model of autism. Neuron. 2014;81(6):1282-9.

19. Kim J-W, Park K, Kang RJ, Gonzales ELT, Kim DG, Oh HA, et al. Pharmacological modulation of AMPA receptor rescues social impairments in animal models of autism. Neuropsychopharmacology. 2019;44(2):314-23.

20. Lazaro MT, Taxidis J, Shuman T, Bachmutsky I, Ikrar T, Santos R, et al. Reduced prefrontal synaptic connectivity and disturbed oscillatory population dynamics in the CNTNAP2 model of autism. Cell Rep. 2019;27(9):2567-78.e6.

21. Jurgensen S, Castillo PE. Selective dysregulation of hippocampal inhibition in the mouse lacking autism candidate gene CNTNAP2.J Neurosci. 2015;35(43):14681-7.

22. Scott R, Sánchez-Aguilera A, van Elst K, Lim L, Dehorter N, Bae SE, et al. Loss of Cntnap2 causes axonal excitability deficits, developmental delay in cortical myelination, and abnormal stereotyped motor behavior. Cereb Cortex. 2017;29(2):586-97.

23. Sacai H, Sakoori K, Konno K, Nagahama K, Suzuki H, Watanabe T, et al. Autism spectrum disorder-like behavior caused by reduced excitatory 
synaptic transmission in pyramidal neurons of mouse prefrontal cortex. Nat Commun. 2020;11(1):5140.

24. Park G, Ryu C, Kim S, Jeong SJ, Koo JW, Lee Y-S, et al. Social isolation impairs the prefrontal-nucleus accumbens circuit subserving social recognition in mice. Cell Rep. 2021;35(6).

25. Bottomley PA. Spatial localization in NMR spectroscopy in vivo. Ann NY Acad Sci. 1987;508:333-48.

26. Tkáć I, Gruetter R. Methodology of H NMR spectroscopy of the human brain at very high magnetic fields. Appl Magn Reson. 2005;29(1):139-57.

27. Tkác I, Starcuk Z, Choi IY, Gruetter R. In vivo 1 H NMR spectroscopy of rat brain at 1 ms echo time. Magn Reson Med. 1999;41(4):649-56.

28. Provencher SW. Estimation of metabolite concentrations from localized in vivo proton NMR spectra. Magn Reson Med. 1993;30(6):672-9.

29. Wilson M, Andronesi O, Barker PB, Bartha R, Bizzi A, Bolan PJ, et al. Methodological consensus on clinical proton MRS of the brain: Review and recommendations. Magn Reson Med. 2019;82(2):527-50.

30. Bolliger CS, Boesch C, Kreis R. On the use of Cramér-Rao minimum variance bounds for the design of magnetic resonance spectroscopy experiments. Neuroimage. 2013;83:1031-40.

31. Alarcón M, Abrahams BS, Stone JL, Duvall JA, Perederiy JV, Bomar JM, et al. Linkage, association, and gene-expression analyses identify CNTNAP2 as an autism-susceptibility gene. Am J Human Genet. 2008;82(1):150-9.

32. Peñagarikano O, Abrahams Brett S, Herman Edward I, Winden Kellen D, Gdalyahu A, Dong H, et al. Absence of CNTNAP2 leads to epilepsy, neuronal migration abnormalities, and core autism-related deficits. Cell. 2011;147(1):235-46.

33. Hitti FL, Siegelbaum SA. The hippocampal CA2 region is essential for social memory. Nature. 2014;508(7494):88-92.

34. Okuyama T, Kitamura T, Roy DS, Itohara S, Tonegawa S. Ventral CA1 neurons store social memory. Science. 2016;353(6307):1536-41.

35. Horder J, Petrinovic MM, Mendez MA, Bruns A, Takumi T, Spooren W, et al. Glutamate and GABA in autism spectrum disorder-a translational magnetic resonance spectroscopy study in man and rodent models. Transl Psychiatry. 2018;8(1):106.

36. Emerson RW, Adams C, Nishino T, Hazlett HC, Wolff JJ, Zwaigenbaum L, et al. Functional neuroimaging of high-risk 6-month-old infants predicts a diagnosis of autism at 24 months of age. Sci Transl Med. 2017;9(393).

37. Chen C-M, Yang P, Wu M-T, Chuang T-C, Huang T-Y. Deriving and validating biomarkers associated with autism spectrum disorders from a largescale resting-state database. Sci Rep. 2019;9(1):9043.

38. Strauss KA, Puffenberger EG, Huentelman MJ, Gottlieb S, Dobrin SE, Parod $J M$, et al. Recessive symptomatic focal epilepsy and mutant contactinassociated protein-like 2. N Engl J Med. 2006;354(13):1370-7.

39. Scott-Van Zeeland Ashley A, Abrahams Brett S, Alvarez-Retuerto Ana I, Sonnenblick Lisa I, Rudie Jeffrey D, Ghahremani D, et al. Altered functional connectivity in frontal lobe circuits is associated with variation in the autism risk gene CNTNAP2. Sci Transl Med. 2010;2(56):56-80.

40. Kurochkin I, Khrameeva E, Tkachev A, Stepanova V, Vanyushkina A, Stekolshchikova E, et al. Metabolome signature of autism in the human prefrontal cortex. Commun Biol. 2019;2(1):234.

41. Ming X, Stein TP, Barnes V, Rhodes N, Guo L. Metabolic perturbance in autism spectrum disorders: a metabolomics study. J Proteome Res. 2012;11(12):5856-62.

42. Peñagarikano $\mathrm{O}$, Geschwind DH. What does CNTNAP2 reveal about autism spectrum disorder? Trends Mol Med. 2012;18(3):156-63.

43. Tristán-Noguero A, Borràs E, Molero-Luis M, Wassenberg T, Peters T, Verbeek MM, et al. Novel protein biomarkers of monoamine metabolism defects correlate with disease severity. Mov Disord. 2021;36(3):690-703.

44. Babkina N, Deignan JL, Lee H, Vilain E, Sankar R, Giurgea I, et al. Early infantile epileptic encephalopathy with a de novo variant in ZEB2 identified by exome sequencing. Eur J Med Genet. 2016;59(2):70-4.

45. Avni E, Ben-Itzchak E, Zachor DA. The presence of comorbid ADHD and anxiety symptoms in autism spectrum disorder: clinical presentation and predictors. Front Psychiatry. 2018;9(717).

46. Tuchman R, Rapin I. Epilepsy in autism. Lancet Neurol. 2002;1 (6):352-8.

47. Simonoff E, Pickles A, Charman T, Chandler S, Loucas T, Baird G. Psychiatric disorders in children with autism spectrum disorders: prevalence, comorbidity, and associated factors in a population-derived sample. J Am Acad Child Adolesc Psychiatry. 2008;47(8):921-9.
48. Oliveira B, Mitjans M, Nitsche MA, Kuo M-F, Ehrenreich H. Excitationinhibition dysbalance as predictor of autistic phenotypes. J Psychiatr Res. 2018:104:96-9.

49. Horder J, Lavender T, Mendez MA, O'Gorman R, Daly E, Craig MC, et al. Reduced subcortical glutamate/glutamine in adults with autism spectrum disorders: a [1H]MRS study. Transl Psychiatry. 2013;3(7):e279.

50. Anitha A, Nakamura K, Thanseem I, Yamada K, Iwayama Y, Toyota T, et al. Brain region-specific altered expression and association of mitochondriarelated genes in autism. Mol Autism. 2012;3(1):12.

51. Bey AL, Wang X, Yan H, Kim N, Passman RL, Yang Y, et al. Brain region-specific disruption of Shank3 in mice reveals a dissociation for cortical and striatal circuits in autism-related behaviors. Transl Psychiatry. 2018;8(1):94.

52. Hoeft F, Carter JC, Lightbody AA, Cody Hazlett H, Piven J, Reiss AL. Regionspecific alterations in brain development in one- to three-year-old boys with fragile X syndrome. Proc Natl Acad Sci. 2010;107(20):9335-9.

53. Gonçalves J, Violante IR, Sereno J, Leitão RA, Cai Y, Abrunhosa A, et al. Testing the excitation/inhibition imbalance hypothesis in a mouse model of the autism spectrum disorder: in vivo neurospectroscopy and molecular evidence for regional phenotypes. Mol Autism. 2017;8(1):47.

54. Poot M. Connecting the CNTNAP2 networks with neurodevelopmental disorders. Mol Syndromol. 2015;6(1):7-22.

55. Fernandes D, Santos SD, Coutinho E, Whitt JL, Beltrão N, Rondão T, et al. Disrupted AMPA receptor function upon genetic- or antibody-mediated loss of autism-associated CASPR2. Cereb Cortex. 2019;29(12):4919-31.

56. Bridi MS, Park SM, Huang S. Developmental disruption of GABA(A)R-meditated inhibition in Cntnap2 KO mice. eNeuro. 2017:4(5).

57. Schmidt MJ, Mirnics K. Neurodevelopment, GABA System Dysfunction, and Schizophrenia. Neuropsychopharmacology. 2015;40(1):190-206.

58. Turrigiano GG, Nelson SB. Homeostatic plasticity in the developing nervous system. Nat Rev Neurosci. 2004;5(2):97-107.

59. Thiagarajan TC, Lindskog M, Malgaroli A, Tsien RW. LTP and adaptation to inactivity: overlapping mechanisms and implications for metaplasticity. Neuropharmacology. 2007;52(1):156-75.

60. Cui Y, Costa RM, Murphy GG, Elgersma Y, Zhu Y, Gutmann DH, et al. Neurofibromin regulation of ERK signaling modulates GABA release and learning. Cell. 2008;135(3):549-60.

61. Silverman JL, Pride MC, Hayes JE, Puhger KR, Butler-Struben HM, Baker $S$, et al. GABAB receptor agonist $R$-baclofen reverses social deficits and reduces repetitive behavior in two mouse models of autism. Neuropsychopharmacology. 2015;40(9):2228-39.

62. Han S, Tai C, Westenbroek RE, Yu FH, Cheah CS, Potter GB, et al. Autisticlike behaviour in Scn1a+/- mice and rescue by enhanced GABA-mediated neurotransmission. Nature. 2012;489(7416):385-90.

63. Ververi A, Vargiami E, Papadopoulou V, Tryfonas D, Zafeiriou DI. Clinical and laboratory data in a sample of Greek children with autism spectrum disorders. J Autism Dev Disord. 2012;42(7):1470-6.

64. Ghaziuddin M, Al-Owain M. Autism spectrum disorders and inborn errors of metabolism: an update. Pediatr Neurol. 2013;49(4):232-6.

65. Cameron JM, Levandovskiy V, Roberts W, Anagnostou E, Scherer S, Loh A, et al. Variability of creatine metabolism genes in children with autism spectrum disorder. Int J Mol Sci. 2017;18(8):1665.

66. Yıldız Y, Göçmen R, Yaramış A, Coşkun T, Haliloğlu G. Creatine transporter deficiency presenting as autism spectrum disorder. Pediatrics. 2020;146(5).

67. Skelton MR, Schaefer TL, Graham DL, deGrauw TJ, Clark JF, Williams MT, et al. Creatine transporter (CrT; Slc6a8) knockout mice as a model of human CrT deficiency. PLOS ONE. 2011;6(1):e16187.

68. Friedman SD, Shaw DW, Artru AA, Richards TL, Gardner J, Dawson G, et al. Regional brain chemical alterations in young children with autism spectrum disorder. Neurology. 2003;60(1):100-7.

69. Corrigan NM, Shaw DWW, Estes AM, Richards TL, Munson J, Friedman SD, et al. Atypical developmental patterns of brain chemistry in children with autism spectrum disorder. JAMA Psychiat. 2013;70(9):964-74.

70. Block W, Jessen F, Träber F, Flacke S, Manka C, Lamerichs R, et al. Regional $\mathrm{N}$-acetylaspartate reduction in the hippocampus detected with fast proton magnetic resonance spectroscopic imaging in patients with alzheimer disease. Arch Neurol. 2002;59(5):828-34. 
71. Hermann D, Sartorius A, Welzel H, Walter S, Skopp G, Ende G, et al. Dorsolateral prefrontal cortex N-Acetylaspartate/total creatine (NAA/tCr) loss in male recreational cannabis users. Biol Psychiat. 2007;61(11):1281-9.

72. Aydin B, Yurt A, Gökmen N, Renshaw P, Olson D, Yildiz A. Trait-related alterations of $\mathrm{N}$-acetylaspartate in euthymic bipolar patients: a longitudinal proton magnetic resonance spectroscopy study. J Affect Disord. 2016;206:315-20.

73. Chaney A, Williams SR, Boutin $\mathrm{H}$. In vivo molecular imaging of neuroinflammation in Alzheimer's disease. J Neurochem. 2019;149(4):438-51.

74. Frye RE, Vassall S, Kaur G, Lewis C, Karim M, Rossignol D. Emerging biomarkers in autism spectrum disorder: a systematic review. Ann Transl Med. 2019;7(23):792.

\section{Publisher's Note}

Springer Nature remains neutral with regard to jurisdictional claims in published maps and institutional affiliations.

- fast, convenient online submission

- thorough peer review by experienced researchers in your field

- rapid publication on acceptance

- support for research data, including large and complex data types

- gold Open Access which fosters wider collaboration and increased citations

- maximum visibility for your research: over 100M website views per year

At BMC, research is always in progress.

Learn more biomedcentral.com/submissions 